\title{
Grid Patient Appointment Template Design to Improve Scheduling Effectiveness
}

\author{
Yu-Li Huang*, Ph.D. and Justin Marcak, MSE \\ Department of Industrial Engineering, New Mexico State University, \\ Las Cruces, NM, USA
}

Submitted April 2014. Accepted for publication October 2014.

\begin{abstract}
Current outpatient delivery systems have been problematic in their ability to effectively schedule appointments and grant patients access to care. A better appointment system has demonstrated improvement on these issues. The objective of this study is to develop a grid appointment system to further improve the scheduling flexibility by determining the minimum length of appointment slots that optimizes the total costs of patient waiting, physician idling, and overtime. This minimum length is used for the patient type requiring the least amount of treatment time such as return visit $(\mathrm{RV})$, and multiplications of the minimum length are for patient types with longer treatment such as new patients (NP). The results indicated that the proposed grid system adjusts to demand changes at least $15 \%$ more cost-effective when grouping two RVs into an NP or dividing an NP into two RVs compared to the base-line scheduling approaches that build around the mean treatment time.
\end{abstract}

Keywords: health services, appointment, healthcare, outpatient scheduling, process improvement

\section{INTRODUCTION}

Patient access to care and long wait times have long been recognized as major problems in modern outpatient delivery systems. It impacts medical staff productivity, stress, quality, and efficiency, as well as health-care cost and availability. The factors that may affect the performance of outpatient delivery systems can vary greatly, such as the clinic environment and condition, set of appointment policies used, punctuality of patients, and demand uncertainty $[1,2]$. To help alleviate negative effects from these factors, a number of methods have been used to determine possible solutions, such as Monte Carlo simulation [3], stochastic modeling [4, 5], dynamic/deterministic optimization models $[6,7]$, and testing different scenarios for a clinical setting through simulation $[8,9]$. Solutions that have been studied to improve outpatient delivery systems include scheduling of resources [10,11], studying forecasting models [12], evaluating patients

*Corresponding author: Yu-Li Huang Ph.D., Department of Industrial Engineering, MSC 4230, New Mexico State University, P.O. Box 30001, Las Cruces, NM 88003 USA, Phone: 575-646-2950, Fax: 575-646-2976. E-mail: yhuang@nmsu.edu. Other author:jbm2005@nmsu.edu. 
before visits [13], the best sequence for scheduling patients [14], predicting patient show or no-show [15], and adjusting appointment times [16]. One of the solutions that is studied by many researchers is redesigning patient appointment templates, also known as appointment rules. These rules determine how a patient may be scheduled based on criteria, needs, and/or resource availability. These rules schedule patients into blocks, where each block is considered an appointment slot and can be used for a single patient or multiple patients [17]. The length of each block is commonly computed using the method of minimizing costs of waiting [18-20] or just waiting itself [21]. Methods that have been used to reduce waiting include determining when an empty appointment slot should be placed [22] and evaluating what ancillary services a patient may need before an appointment [23, 24]. Rather than a fixed length for each block, some researchers studied another way of determining patient scheduling, called a Dome Pattern scheduling scheme. This scheduling scheme works off of the concept that appointment slot times gradually increase through to the middle of a clinic session and then gradually decrease towards the end of the session to alleviate the effects of physician lateness and service interruption [16] and the negative impacts from noshows and cancellations [25]. However, in reality, the most common existing method we have seen to decide the block size is to apply the average value of physician treatment time, which is presumably to be the easiest way rather than adopting a complex algorithm. Once the number of blocks and their size are determined, patients are scheduled according to their needs and conditions. Patients can also be scheduled through a combination of scheduling rules [26]. The block scheduling systems are by far the most commonly used methods.

Another method that is especially popular in primary care is the open-access scheduling. Open-access is a concept that tries to schedule appointments for patients during a relatively short scheduling horizon to prevent patients from becoming a noshow. However, implementation can actually be difficult - especially for patients that wish to obtain appointments [27-29]. This concept proposes to fulfill daily demand regardless of clinic capacity which could be effective in meeting patients' needs for accessing appointments [30], accommodating walk-ins, and reducing the negative impact of no-shows and cancellations [31], but could potentially burden clinics and increase patient wait time [32]. One of the key issues that have not been studied to the best of our knowledge is the flexibility of appointment scheduling slots. Many researchers have conducted studies that tried to achieve so-called "flexible" scheduling, but they are mostly algorithms that meet specific constraints according to facilities and resources, such as the scheduling of physicians that meets federal guidelines and when physicians prefer to have breaks [33-35]. Flexibility should be a concept that allows an adjustment to an appointment scheduling that does not potentially incur extra costs or change resource requirements, such as the change in the length of appointment slots to meet the needs of patients without generating additional waiting for both patients and physicians. Most appointment templates consist of multiple patient types and the slot length of each patient type varies depending on physician treatment time [21]. The number of slots for each patient type is estimated according to the forecast of demand [36-38]. Although patient demand fluctuates daily, the common practice is to either 
keep the appointment slots to be the same or to develop a scheduling scheme that accommodates these fluctuations, in that there are extra slots created for last minute appointments [30]. Rather than dealing with daily demand changes for different types of patients, some studies proposed to have a fixed-length appointment slot for all patients regardless of the procedure needed [39]. This fixed-length appointment slot for all patient types essentially embeds the high variation or uncertainty for physician treatment time, which normally leads to higher patient wait time and physician idle time [40]. As for the majority of clinics with schedules that have at least two patient types such as new patients and return visit patients [41]. When patient demands come, they incline to schedule patients into the appointment slots that are not designed for their treatment duration, such as scheduling a return visit patient into a new patient slot or vise versa. This creates the problem of waiting for both patients and physicians, which could potentially lead to significant overtime.

In short, the block system is most effective when patients are scheduled into their designed appointment slots and the open access approach may be able to adjust to daily patient demand, but will encounter long patient wait time and overtime. Some clinics have used systems that require the minimum grid size. If the minimum grid is 15 minutes, then for patients requiring longer treatment times will have to take an increment of 15 minutes such as 30 or 45 minutes. The commonly used minimum grid is either 10 or 15 minutes, supposedly, because of the assumption of average physicians' treatment time. These systems using the minimum grid are certainly more flexible to accommodate daily demand by combining or splitting appointment slots [42]. The question is how the minimum grid is determined. Therefore, this paper proposes to develop a unique approach to determining the minimum grid that optimizes the total cost of patient wait time, physician idle time, and overtime. The proposed minimum grid system would not only be cost-effective but also provide flexibility to accommodate the needs of daily patient demand.

\section{METHODS}

The minimum grid is designed for the patient type with the least amount of treatment time. The other longer treatment patient types will be given their treatment length in increment of the minimum grid. By doing this, the scheduling template is able to combine or divide slots to meet the demand so that the length of treatment time for different patient types is not compromised.

\subsection{Model Development}

The basic patient flow was developed to account for the concept of minimum grids for simulation purposes. The model definitions and formulations are the following:

$T_{i, j, k}=$ physician service time for patient $k$ from group $j$ at time slot $i$, where $i=1,2$,

$3, \ldots, n, j=1,2,3, \ldots m$, and $k=1,2,3, \ldots, q$.

$n=$ the total number of scheduling time slots per clinic session.

$m=$ the number of patient groups. For example, the patient groups could be new patient, return visit, post operation.

$q=$ the number of patients scheduled in a time slot. 
$T_{i, j, k} \sim G\left(\mu_{j}, \sigma j^{2}\right), G$ is a probability distribution with mean $\mu_{\mathrm{j}}$ and standard deviation $\sigma_{j} . T_{i, j, k}$ consists of times for all works required for a physician to serve a patient, including preparation time, patient encounter time, and charting time.

$$
T_{i, j, k}= \begin{cases}T_{i, j, k} & 1-r_{i, k}(\text { the patient } k \text { shows up at time slot } i) \\ 0 & \text { Otherwise }\end{cases}
$$

$r_{i, k}=$ the no-show rate for the patient $\mathrm{k}$ at time slot $i$.

$X_{\min }=$ the minimum grid (time interval) for a slot in minutes. Normally, the minimum grid is based on the return visit patient group since they require the least amount for treatment time. The time interval for other patient types will be a multiplication of the minimum grid. Assuming patient groups are sorted based on increasing order of their treatment time, the $X_{\min }$ is from the first patient group and can also be denoted as $X_{1}$. The next time interval for the second patient group is $X_{2}=2 X_{\min }$. The time interval for the third patient scheduled group is $X_{3}=3 X_{\min }$. Therefore, in general, the time interval for group $j$ is $X_{j}=j X_{\min }$.

The designed starting time is determined as:

$$
S_{i, j, k}=S_{i-1, j, k}+X_{j}, i \geq 2
$$

where $S_{i, j, k}=$ the designed starting time for the patient $k$ from group $j$ at time slot $i$, and let $S_{i, j, k}=0$. Only the patients from the same patient group can be overbooked at the same time slot.

Assuming punctual patient arrival, the physician finish time is determined as:

$$
F_{i, j, k}=A_{i, j, k}+T_{i, j, k}
$$

where $F_{i, j, k}=$ the physician finish time with the patient $k$ from group $j$ at time slot $i$.

$A_{i, j, k}=$ the actual service start time for the patient $k$ from group $j$ at time slot $i$.

$$
A_{i, j, k}= \begin{cases}S_{i, j, k} & \text { if } F_{i, j, k-1}=S_{i, j, k} \text { or } F_{i-1, j, q} \leq S_{i, j, k} \\ F_{i, j, k-1} & \text { if } F_{i, j, k-1}>S_{i, j, k} \\ F_{i-1, j, q} & \text { Otherwise }\end{cases}
$$

The patient's wait time is:

$$
W_{i, j, k}=A_{i, j, k}-S_{i, j, k}
$$

where $W_{i, j, k}=$ the wait time for the patient $k$ from group $j$ at time slot $i$. 
The total patient wait time $W$ is:

$$
W=\sum_{i=1}^{n} \sum_{k=1}^{p} W_{i, j, k}
$$

The physician idle time waiting for the patient is determined from eqns. 2 and 3:

$$
P_{i, j, k}=S_{i, j, k}-F_{i-1, j, q}
$$

where $P_{i, j, k}=$ the physician idle time waiting for the patient $k$ from group $j$ at time slot $i$.

The total physician idle time $P$ can be determined based on eqn. 7:

$$
P=\sum_{i=1}^{n} P_{i, j, k}
$$

The overtime for a clinic day is determined as:

$$
O=F_{n, j, q}-E
$$

where $O=$ overtime for a clinic day, and $E$ is the expected finish time in a clinic day

Let $c_{w}$ be the cost of patient wait time per minute, $c_{P}$ be the cost of physician idle time per minute, and $c_{o}$ be the cost of overtime per minute, then the total cost of patient wait time, physician idle time and overtime $\left(c_{T}\right)$ is:

$$
c_{T}=c_{w} W+c_{p} P+c_{o} O
$$

The objective is to find the minimum grid $\left(X_{\min }\right)$ that minimizes the expected value of $c_{T}$.

\subsection{Example Result from Simulation Optimization}

The simulation was modeled using a Microsoft Excel macro function applied to a hypothetical physician's daily clinic schedule as an example to demonstrate the use of the method. The model is based on that (1) all patients who arrived would be on time; (2) inter-appointment time such as time spent on non-clinical activities was not considered; (3) two different patient types included were new patient (NP) and return visit patient (RV) and let $j=1$ for RV and 2 for NP; (4) the physician service time is assumed to be $T_{i, j, k} \sim \operatorname{Gamma}\left(15,10^{2}\right), T_{i, 2, k} \sim \operatorname{Gamma}\left(30,20^{2}\right)$; (5) there are a total of 24 patient slots ( $8 \mathrm{NP}$ and $16 \mathrm{RV}$ ) distributed evenly throughout a clinic day; (6) the costs were $c_{w}=10, c_{p}=50, c_{o}=75$ in $\$$ per hour; (7) no show rate is assumed 
as $r_{i, k}=0.15$; (8) no overbooking and expected finish clinic time in a 8-hour day from 8:00 am to $4: 00 \mathrm{pm}$ for a total of 480 minutes $(E=480)$ without lunch break. The goal is to find the minimum grid, $X_{\min }$, that minimizes the estimate of the total cost of $\mathrm{c}_{\mathrm{T}}=c_{w} W+c_{p} P+c_{o} O$ assuming a set of scheduling templates (see Table 1). To be noted, the scheduling patient sequence changes according to patient type demand distributions, which change the number of patients scheduled in a clinic day (see section 3.3). Right-skewed distributions such as Lognormal distribution [43, 44], Gamma distribution [45, 46], and Exponential distribution [18] are commonly used to model physician treatment time. For the length of appointment slots, $X_{1}=X_{\min }$ is for the RV slot and $X_{2}=2 X_{\min }$ is for NP. A simulation optimization technique is used to find the minimum grid from 100 simulation runs that are produced. Each simulation run represents a possible scenario for a clinic day. Hence, our simulation optimization is representative of a Monte Carlo simulation technique that runs

Table 1. An example of a set of scheduling template

\begin{tabular}{lccc}
\hline $\begin{array}{l}\text { Appointment } \\
\text { Slot }(\boldsymbol{i})\end{array}$ & Patient Type & $\begin{array}{c}\text { Slot Length at } \boldsymbol{X}_{\text {min }}= \\
\text { 14 minutes }\end{array}$ & $\begin{array}{c}\text { Appointment } \\
\text { Time }\end{array}$ \\
\hline 1 & RV & 14 & $8: 00$ \\
2 & RV & 14 & $8: 14$ \\
3 & NP & 28 & $8: 28$ \\
4 & RV & 14 & $8: 56$ \\
5 & RV & 14 & $9: 10$ \\
6 & NP & 28 & $9: 24$ \\
7 & RV & 14 & $9: 52$ \\
8 & RV & 14 & $10: 06$ \\
9 & NP & 28 & $10: 20$ \\
10 & RV & 14 & $10: 48$ \\
11 & NP & 28 & $11: 02$ \\
12 & RV & 14 & $11: 30$ \\
13 & RV & 14 & $11: 44$ \\
14 & RV & 14 & $11: 58$ \\
15 & NP & 28 & $12: 12$ \\
16 & RV & 14 & $12: 40$ \\
17 & RV & 14 & $12: 54$ \\
18 & NP & 28 & $1: 08$ \\
19 & RV & 14 & $1: 36$ \\
20 & NP & 28 & $1: 50$ \\
21 & RV & 14 & $2: 18$ \\
22 & RV & 14 & $2: 32$ \\
23 & NP & 28 & $2: 46$ \\
24 & RV & 14 & $3: 14$ \\
\hline & & &
\end{tabular}


multiple trials to converge the solution. The simulation optimization algorithm is the following:

Step 1: Select grid size

The initial grid size $(X)$ should be selected around the mean service time of the patient type that requires the least amount of physician service time. The appointment slot length for patients requiring longer service time will be scheduled at the integer multiplication of $X$. For the example demonstrated here, the slot length for RV should be $X$ and the length for NP should be $2 X$.

\section{Step 2: Find the total cost}

Once the grid size is chosen, the simulation of a clinic flow with 100 replications is run to compute the expected total $\operatorname{cost} c_{T}(X)$. Let $c_{T}(X)$ be the total cost estimated by the minimum grid size $X$.

\section{Step 3: Repeat Step 1 and Step 2}

Repeat Steps 1 and 2 for a grid size of increment (or decrement) of one minute from the selected grid size and compute the total cost. $X$ takes an integer value in minutes, i.e., 12 minutes, for the convenience of scheduling systems.

\section{Step 4: Total Cost Comparison}

Compare the total cost of $c_{T}(X)$ to $c_{T}(X+1)$ or $c_{T}(X-1)$. This step is to determine the direction to the minimum cost. If $c_{T}(X)>c_{T}(X+1)$, then the selected grid size becomes $X+1$; if $c_{T}(X)>c_{T}(X-1)$, then the selected grid size becomes $X-1$. Once the direction is determined (either increment or decrement of the selected grid size), the one-minute increment (or decrement) of the grid size will be continue trialed by repeating steps 2, 3, and 4 until the stopping criteria is reached. The optimal solution search stops when $c_{T}(X)<c_{T}(X+1)$ and $c_{T}(X)<c_{T}(X-1)$. Then this $X$ is considered to be $X_{\min }$.

The simulations resulted in that the total cost of patient wait time, physician idle time, and overtime are minimized at 182 where the minimum grid size $\left(X_{\min }\right)$ is 14 minutes (See Figure 1). Therefore, the patient appointment time for the given set of scheduling templates is made according to 14 minutes for RV and $28(2 \times 14)$ minutes for NP, as shown in Table 1.

\section{RESULTS}

The proposed minimum grid scheduling system has two advantages over the other scheduling methods. It is cost-effective in terms of patient wait time, physician idle time, and overtime. It is also flexible or interchangeable among appointment slots of different patient types to meet the demand. To further demonstrate the effectiveness, comparison of cost and interchangeability are conducted with some base-line scheduling approaches through simulations. The base-line scheduling methods used to compare with the proposed minimum grid scheduling (PS) are:

Single block $(\mathrm{SB})$ - patients are scheduled in individual blocks at fixed intervals of the mean treatment time. 


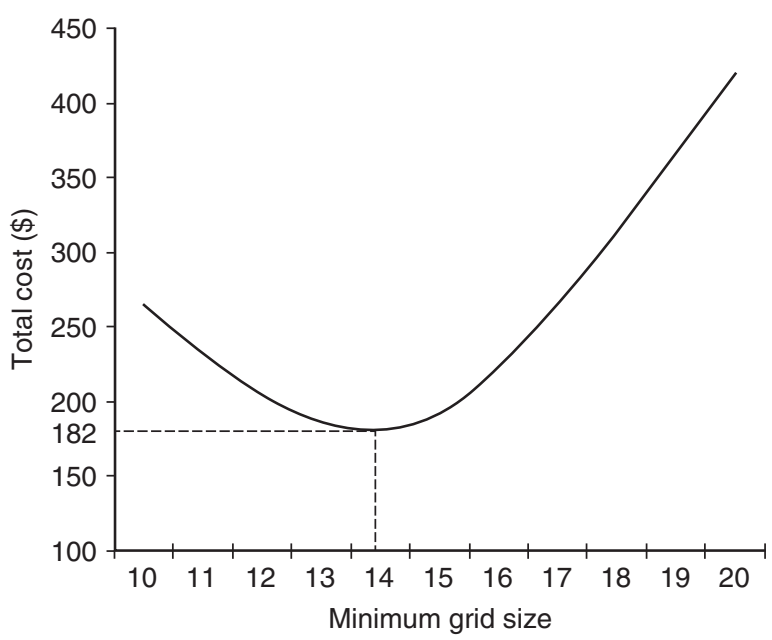

Figure 1. The optimal total cost of $\$ 182$ occurs when minimum grid $X_{\min }=$ 14 minutes.

Double block (DB) - two patients are scheduled at the same time at fixed intervals that sums up the mean treatment times.

Single block with an initial double book (SI) - patients are scheduled in individual blocks at fixed intervals of the mean treatment time with two patients in the first block. The method is to prevent physician from idling in the beginning of a clinic session when the first patient does not show.

Dome scheduling (DS) - the first and last quarters of individual blocks at the fixed interval of less than the mean treatment time and the second and third quarters of blocks at the fixed interval of longer than the mean treatment time. This is similar to "plateaudome' method [16]. To simplify this scheduling method, the first and last quarters are one minute less than the mean treatment time and the second and third quarters are one minute more than the mean treatment time to ensure that patients are scheduled within an 8-hour clinic day.

\subsection{Cost Effectiveness}

To illustrate the cost-effectiveness of this proposed approach, the comparison between the proposed minimum grid approach and a common approach using mean treatment time as the length of appointment slots is conducted. Key factors for designing scheduling templates were incorporated to ensure that the cost effectiveness was across the board of different scenarios. For simplicity, only two patient types, NP and RV, are considered. The key factors considered are: treatment time ratio between NP and RV, cost ratio between physician idle time, and patient wait time, treatment time variation, 
no-show rate, and the demand for each patient type. The detail descriptions of each factor are:

- Treatment time ratio between NP and RV is used with the primary purpose to demonstrate the treatment time difference in terms of a ratio between NP and RV. Five levels of ratios are considered here: 1.5, 1.75, 2, 2.25, and 2.5. This means if RV treatment time is 15 minutes on average, then NP treatment times are $22.5,26.25,30,33.75$, and 37.5 minutes, respectively. Since the length of $\mathrm{NP}$ and RV treatment times are different by clinics and specialties, these five levels should demonstrate the range difference to generalize the impact of this factor. For simulation purposes, Gamma distribution is used for treatment time distribution.

- Cost ratio of physician idle and patient wait time $\left(c_{P} / \mathrm{c}_{\mathrm{w}}\right)$ is taken at five levels: 1 , $3,5,10$, and 20 , assuming $\$ 10$ per hour for patient wait time and overtime is 1.5 times the physician's idle cost. The physician idle time costs are $\$ 10, \$ 30, \$ 50$, $\$ 100$, and $\$ 200$, respectively. The cost ratios of physician idle and patient wait time are different between physicians and among specialties. These five levels should be sufficient to indicate the differences from low to high cost ratios.

- Treatment time variation is analyzed by coefficient of variation $(\mathrm{CV}) . \mathrm{CV}$ is an indication of the relative ratio between variation and mean of the treatment time. Five levels are adopted: $1,0.8,0.67,0.57$, and 0.5 .

- No-show rates are taken at three levels: $5 \%, 15 \%$, and $30 \%$. There are many other factors that contribute to patient no-show and it differs by clinics and specialties. These three levels should cover the range of no-show rates in majority of clinics.

- Demands for patient types at three different scenarios are considered. A total of 24 patients in a clinic day are assumed. The first scenario consists of $8 \mathrm{NP}$ and 16 RV slots. The second scenario has 6 NPs and 18 RVs. The third scenario includes 4 NPs and 20 RVs. The slots are distributed evenly throughout a day. These three different scenarios represent the demand difference among clinics and how they impact the scheduling template design. The three scenarios are shown in Table 2 .

There were a total of 1125 combination cases presented and 100 simulations were run for each case. They were run through the proposed minimum grid approach (PS) and other base-line approaches. The analysis of variance (ANOVA) is used to analyze whether the proposed approach (PS) is superior to other base-line approaches at $\alpha=0.05$. The ANOVA result, shown in Table 3, indicates that the proposed minimum grid system is significantly lower in total cost than the other approaches. The average cost is reduced, compared to the minimum grid approach (PS), by $21.8 \%, 24.7 \%, 15.6 \%$, and $21.8 \%$ when compared to single block (SB), double block (DB), single block with initial overbook (SI), and dome scheduling (DS), respectively. The maximum cost saving is 54.7\%, $49.8 \%$, $49.8 \%$, and $55.2 \%$, respectively, for the scenario with most NP slots, the highest NP/RV treatment time ratio, the highest cost ratio, the highest coefficient of variation, and the highest no-show rate. A box plot is demonstrated in Figure 2 for visual comparison.

ANOVA results also reveal the impact from each factor on the total cost. When there are more NP slots, there is a higher NP/RV ratio, cost ratio, coefficient of variation, and lower no-show rate that results in a higher cost. Having more high treatment slots such as NP means the overall treatment time is longer which leads to the higher chances of going overtime and a higher cost on patient wait time. The same trend as to the higher 
248 Grid Patient Appointment Template Design to Improve Scheduling Effectiveness

Table 2. Three different scenarios of demand for each patient type

\begin{tabular}{lccc}
\hline Patient Slots & Scenario 1 & Scenario 2 & Scenario 3 \\
\hline 1 & RV & RV & RV \\
2 & RV & RV & RV \\
3 & NP & NP & RV \\
4 & RV & RV & NP \\
5 & RV & RV & RV \\
6 & NP & RV & RV \\
7 & RV & NP & RV \\
8 & RV & RV & NP \\
9 & NP & RV & RV \\
10 & RV & NP & RV \\
11 & NP & RV & RV \\
12 & RV & RV & RV \\
13 & RV & RV & RV \\
14 & RV & NP & RV \\
15 & NP & RV & RV \\
16 & RV & RV & NP \\
17 & RV & RV & RV \\
18 & NP & NP & RV \\
19 & RV & RV & RV \\
20 & NP & RV & NP \\
21 & RV & RV & RV \\
22 & RV & NP & RV \\
23 & NP & RV & RV \\
24 & RV & & RV \\
\hline & & &
\end{tabular}

Table 3. ANOVA results of 1125 different scenarios for total cost

\begin{tabular}{lccccc}
\hline Source & $\boldsymbol{D F}$ & $\boldsymbol{S S}$ & $\boldsymbol{M S}$ & $\boldsymbol{F}$-test & $\boldsymbol{P}$-value \\
\hline approach & 4 & 287930000 & 71983000 & 2832.4 & $<0.0001$ \\
treatment time ratio & 4 & 1250700000 & 312680000 & 12303 & $<0.0001$ \\
cost ratio & 4 & 9341500000 & 2335400000 & 91893 & $<0.0001$ \\
treatment time variation & 4 & 1106200000 & 276550000 & 10882 & $<0.0001$ \\
no-show rate & 2 & 29765000 & 14882000 & 585.6 & $<0.0001$ \\
demand & 2 & 846660000 & 423330000 & 16657 & $<0.0001$ \\
residuals & 562479 & 14295000000 & & & \\
total & 562499 & & & & \\
\hline
\end{tabular}




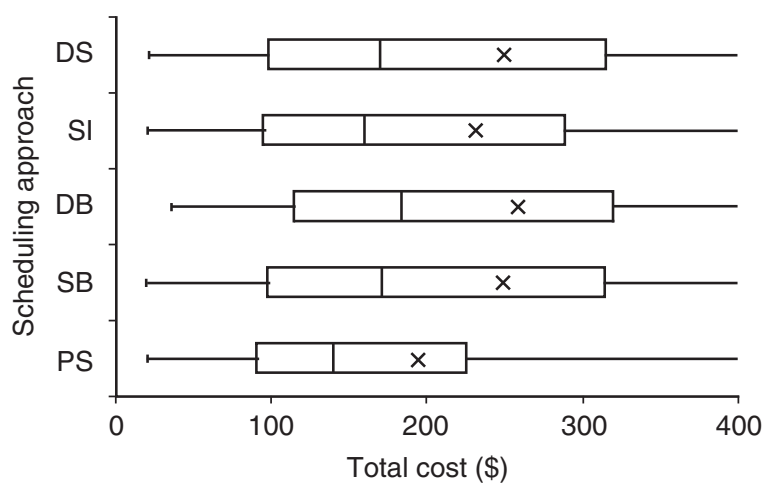

Figure 2. Box plot for approach comparison in total cost where $\times$ represents the average.

NP/RV treatment time ratio means longer NP treatment time. This will certainly increase higher patient wait and chances of overtime. The high cost ratio implies that there are specialty clinics. When the cost of a physician's time is higher, the overtime cost is also higher accordingly. For the no-show rate, since the high no-show rate creates uncertainty of patient flow, the impact of total cost could go either way. It could benefit less patient wait time and lower the chances of overtime, but physician idle time may be increased. In terms of $\mathrm{CV}$, the higher $\mathrm{CV}$ generates higher uncertainty of treatment that could either lead to higher treatment times which increases patient wait time and overtime or lead to lower treatment time that produces physician idle time. In either case, the total cost is increased.

\subsection{Appointment Slot Interchangeability}

The uniqueness of the proposed minimum grid system is to be able to either combine or divide appointment slots to meet daily demand without compromising patient wait time and clinic resources. The interchangeability of appointment slots was compared between the proposed and the base-line approaches. The demand for the second scenario, which consists of 6 NPs and $18 \mathrm{RVs}$, was studied. The comparison was conducted based on cases of combining 4 RVs into 2 NPs and dividing 2 NPs into 4 RVs. Let $\mu_{j}$ be the average treatment time for patient type $j$. Combining $2 \mathrm{RVs}$ $(j=1)$ into a NP $(j=2)$, if $2 \mu_{1}<\mu_{2}$, then patient delay could be generated and compounded for the rest of clinic day, or if $2 \mu_{1}>\mu_{2}$, then physician idle could occur. For dividing an NP into $2 \mathrm{RVs}$, if $1 / 2 \mu_{2}<\mu_{1}$, patient wait would likely occur. On the other hand, if $1 / 2 \mu_{2}>\mu_{1}$, then physician idle is inevitable. The proposed grid system accounts for these problems in developing a minimum grid for accommodation.

In the case of combining $2 \mathrm{RVs}$ into an NP, two sets of consecutive RVs were selected from scenario 2: slots 5 and 6 , slots 17 and 18 . Two NP slots were created for a total of 22 patients in a clinic day. The same level of factors of treatment time ratio, cost ratio, treatment time variation, and no-show rate were also considered for 
comparison. The ANOVA result indicated that the proposed grid system outperformed the base-line approaches in total cost at a significance level of 0.05 . The proposed approach (PS) has a total cost of $\$ 190.4$ per clinic day that is $21.9 \%, 15.1 \%, 24.7 \%$, and $21.7 \%$ lower than the single block (SB), the single block with initial overbook (SI), the double block (DB), and the dome scheduling (DS), respectively. The maximum cost saving from 100 simulation runs is $48.1 \%, 42.1 \%, 44.4 \%$, and $48.5 \%$ as compared to the base-line approaches at the highest NP/RV treatment time ratio, highest cost ratio, highest coefficient of variation, and highest no-show rate.

For the case of dividing an NP into two RVs, two NPs were selected from scenario 2: slot 7 and slot 19. Four RV slots were created for a total of 26 patients in a clinic day. The same level of factors of treatment time ratio, cost ratio, treatment time variation, and no-show rate were taken into account for comparison. The ANOVA result also indicated that the proposed grid system has a significant lower total cost than the common approach. The proposed approach has a total cost of $\$ 193.3$, that is $20.2 \%, 14.1 \%, 22.3 \%$, and $20.1 \%$ lower than SB, SI, DB, and DS, respectively. The maximum cost saving is $50.9 \%, 44.9 \%, 46.4 \%$, and $51.6 \%$ in comparison to the base-line approaches at the highest $\mathrm{NP} / \mathrm{RV}$ treatment time ratio, highest cost ratio, highest coefficient of variation, and highest no-show rate.

\subsection{Case Study}

A real-life case study for a physician in a primary care clinic is presented here to first demonstrate the feasibility of our proposed approach and then to evaluate its effectiveness over the existing scheduling scheme. The physician is currently seeing five types of patients: follow up (FU), new patient (NP), office visit (OV), physical exam (PE), and well child care (WCC). The service times (mean, standard deviation) including preparation and charting times in minutes are $(24.8,12.1),(39.6,8.4)$, $(24.6,10.7),(37.2,10.8)$, and $(19.5,8.6)$, respectively. The patient type demand distributions are $32.3 \%, 3.8 \%, 55.6 \%, 3.8 \%$, and $4.5 \%$. The clinic currently schedules the last patient at 11:40 am in the morning (starting at $8 \mathrm{am}$ ) and 4:40 pm in the afternoon (starting at $1 \mathrm{pm}$ ). The existing scheduling scheme has two different scheduling time slots, 20 or 40 minutes. Patients who are FU and OV are scheduled in 20-minute slots and patients who are classified as NP, PE, and WCC are scheduled in 40-minute slots. The current no-show rate is at $21 \%$. A simulation model is built using the proposed minimum grid concept, assuming service times follow Gamma distribution, no physician lateness, punctual patient arrival, and cost of patient wait time is $\$ 10$ per hour, physician idle time is $\$ 50$ per hour, and clinic overtime is $\$ 75$ per hour. One hundred simulations were run. Each simulation represents a possible clinic day. The number of patients seen in a clinic day is adjusted to patient type distributions. The maximum number of patients that can be scheduled in a clinic day are 22 patients where all patients are identified as 20-minute patients. The minimum number of patients that can be scheduled are 12 patients that consist of five 40-minute patients and a 20-minute patient in the morning and the afternoon. The simulation optimization results indicate that the scheduling scheme should have a minimum grid of 18 minutes, rather than the current 20 minutes, where the expected 


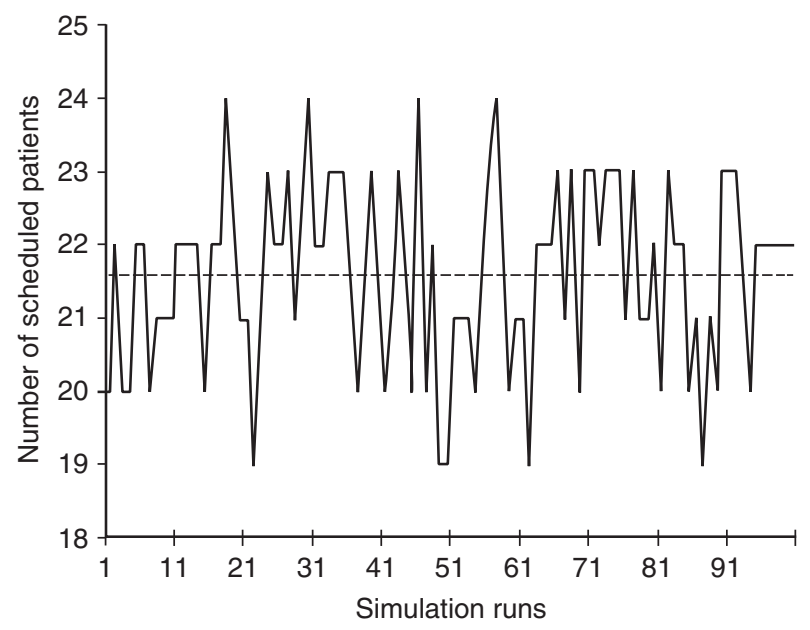

Figure 3. The number of patients scheduled for each simulation run in the case study.

total cost is minimized. The total expected cost for the proposed $18 / 36$ schedule is at $\$ 159$ per clinic day and for the current 20/40 schedule is at $\$ 167$. This clinic has been using the concept of the minimum grid system. However, the determination of the minimum grid is arbitrarily chosen; perhaps it is more convenient without actually collecting data on physician's service time.

\subsubsection{Number of Patients Scheduled Accounting for Demand Uncertainty}

Through the case study, the demand distributions for each patient type were considered to determine the minimum grid size. The number of patients scheduled depended on the appointment slot time needed (either 20 or 40 minute appointments) and the total scheduled time for the clinic session needed to be 480 minutes or less. Figure 3 indicates that the average number of patients scheduled from 100 simulation runs was 21.6 with the maximum of 24 and the minimum of 19 patients. Each simulation run represented a possible clinic day. The total cost of each run was computed and the overall expected cost was calculated from 100 runs to conclude the minimum grid size.

\section{DISCUSSION}

\subsection{Impact of Factors on the Minimum Grid}

The proposed minimum grid system considered the treatment time difference among patient types and demonstrated the efficiency in total cost of patient wait time, physician idle time and overtime. In addition, it also provided a cost-effective way of interchanging appointment slots to meet daily demand. To understand how the minimum grid changes according to clinical differences, the following discussion is conducted in terms of the factors introduced in Section 3.1. The factors are treatment time ratio between NP and RV, cost ratio between patient wait time and physician idle 
252 Grid Patient Appointment Template Design to Improve Scheduling Effectiveness

time, the size of treatment time variation, patient no-show rate, and the demand for appointments. After 100 simulation runs for each scenario (1125 scenarios in total), the ANOVA results indicated that all factors significantly impact the determination of the minimum grid at a level of 0.05 ; see Table 4 .

Table 4. ANOVA results of 1125 different scenarios for the minimum grid

\begin{tabular}{lccccc}
\hline Source & $\boldsymbol{D F}$ & $\boldsymbol{S S}$ & $\boldsymbol{M S}$ & $\boldsymbol{F}$-test & $\boldsymbol{P}$-value \\
\hline treatment time ratio & 4 & 56764 & 14191 & 40876.8 & $<0.0001$ \\
cost ratio & 4 & 537343 & 134336 & 386950.7 & $<0.0001$ \\
treatment time variation & 4 & 215 & 54 & 155.2 & $<0.0001$ \\
no-show rate & 2 & 178851 & 89425 & 257587.9 & $<0.0001$ \\
demand & 2 & 8346 & 4173 & 12020.7 & $<0.0001$ \\
residuals & 112483 & 39050 & 0.35 & & \\
total & 112499 & 820569 & & & \\
\hline
\end{tabular}
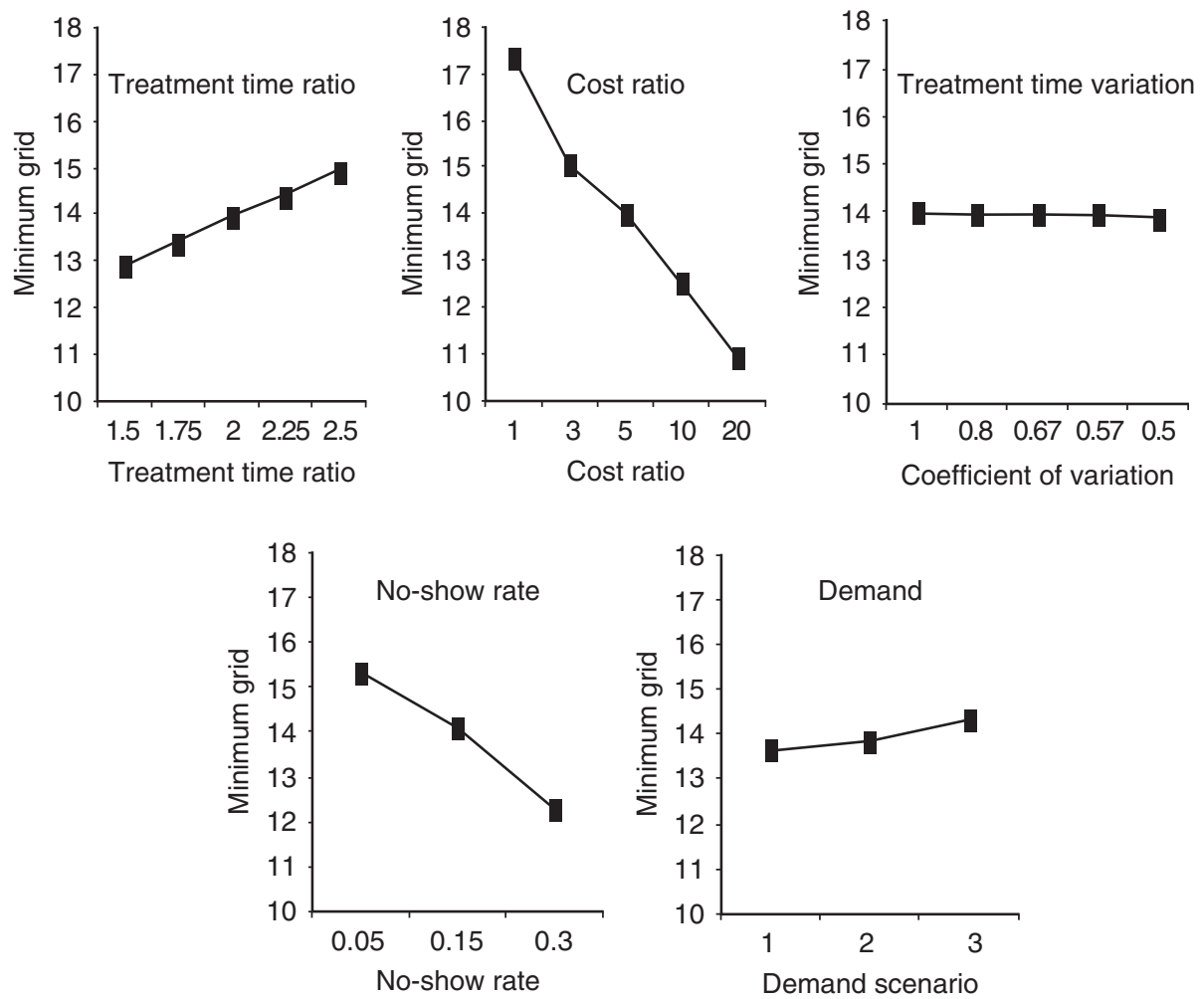

Figure 4. The impact of individual factors on the determination of the minimum grid. 
The minimum grid $\left(X_{\min }\right)$ system adjusts to each scenario. In general, $X_{\min }$ increases when treatment time ratio and variation increase and when cost ratio, no-show rate, and demand of NP decrease, as shown in Figure 4. Figure 4 indicates that cost ratio has the most significant impact on the changes of the minimum grid, while the treatment time variation has the least impact. The increase of treatment time ratio and variation means the uncertainty of treatment time increases. In order to accommodate the uncertainty, a larger $X_{\min }$ is necessary. On the other hand, the higher cost ratio means that physician idle time is valued higher than patient wait time. To prevent a physician from idling, $X_{\min }$ would have to be smaller to ensure that the next patient will always be in the exam room before the physician finishes the current patient. In terms of no-show rate, the higher no-show rate means that the patient flow could be more relaxed since the no-show appointment slots often serve as a buffer for physicians to catch up with their clinic flow. In this case, the smaller $X_{\min }$ would help eliminate the waiting from both patients and physicians. As for the demand, it is very intuitive that the higher NP demand means the clinic has a tendency to run longer since NP would normally take a longer time than RV. In order for a clinic to finish on time and prevent overtime, a smaller $X_{\min }$ is adjusted since overtime incurs the highest cost.

The minimum grid ranges from 8 to 23 minutes in 1125 scenarios. To further understand the impact of each factor, a decision tree analysis was conducted. The decision tree was done using $R$ 2.12.1 version statistical software. For simplicity of the analysis, let TR be the treatment time ratio, CR be the cost ratio, NS be the no-show rate, $\mathrm{CV}$ be the treatment time variation, and $\mathrm{D}$ be the demand. The purpose is to discuss how the minimum grid decision is classified and extrapolated based on these factors. Figure 5 demonstrated the decision tree results in detail and graphical formats.

The detail decision tree features the splits with the count of scenarios, deviance, and the value of the minimum grids. The asterisk indicated the end node for the classification of the minimum grids. The results provide the insight of how 1125 scenarios are sliced

1) root 11258205.6920013 .945780

2) $\mathrm{CR}>=7.54501568 .0980011 .702220$

4) $\mathrm{NS}>=0.225150 \quad 172.673309 .713333$

8) $\mathrm{CR}>=157533.520008 .920000 *$

9) $\mathrm{CR}<157544.7466710 .506670 *$

5) NS $<0.225300505 .3967012 .696670$

10) $\mathrm{CR}>=15150170.1933011 .926670 *$

11) $\mathrm{CR}<15150157.3333013 .466670 *$

3) $\mathrm{CR}<7.56752862 .4390015 .441480$

6) $\mathrm{CR}>=24501016.4980014 .497780$

12) NS $>=0.225150167 .7600013 .040000 *$

13) NS $<0.225300370 .5867015 .226670$

26) $\mathrm{TR}<1.875120104 .0000014 .500000 *$

27) $\mathrm{TR}>=1.875180160 .9778015 .711110 *$

7) $\mathrm{CR}<2225643.6622017 .328890$

14) NS $>=0.2257555 .9466716 .026670 *$

15) $\mathrm{NS}<0.225150396 .9400017 .980000$

30) $\mathrm{TR}<2.12590 \quad 114.1000017 .100000 *$

31) $\mathrm{TR}>=2.12560108 .6000019 .300000 *$

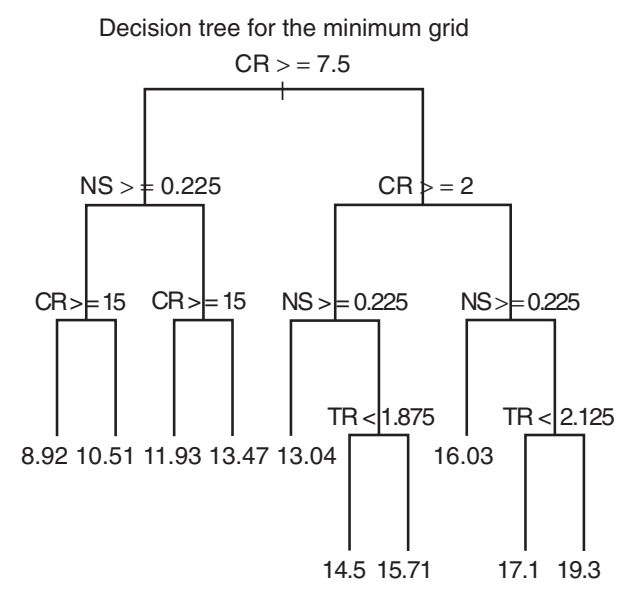

Figure 5. The decision tree results in detail (left) and graphical (right) formats. 
by these factors. Only three factors are used for the significant splits, i.e., CR, NS, and TR, since they have the most significant impact. The first significant split is done by CR. To be classified with the minimum grid of 12 minutes, the condition should be CR $\geq 7.5$, $\mathrm{NS}<0.225$, and $\mathrm{CR} \geq 15$. For the minimum grid to be about 16 minutes, the classification should be $\mathrm{CR}<7.5, \mathrm{CR} \geq 2, \mathrm{NS}<0.225$, and $\mathrm{TR} \geq 1.875$. This discussion helps clinics to be able to predict the minimum grid size and understand the magnitude of minimum grid changes due to the changes of clinic conditions.

\subsection{Implementation of the Minimum Grid System}

The implementation of the minimum grid system is relatively straightforward. Often in a real clinic setting, the physician or the scheduler cannot know the sequence of the request for appointment slots in advance. Continuing with the case study from section 3.3 , the total cost was optimized when the minimum grid was 18 minutes, which generates a scheduling scheme of 18/36. The clinic will first set up the daily scheduling template starting from 8:00 am in the morning and 1:00 pm in the afternoon with an 18-minute increment. The schedule will look like 8:00, 8:18, 8:36, 8:54, 9:12, 9:30, 9:48, and so on. The last slot will be scheduled at 11:18 am in the morning and at 4:18 $\mathrm{pm}$ in the afternoon for a total of 24 slots. When a scheduler receives a request for an appointment, he/she needs to first identify if the patient requires 18 minutes or 36 minutes. If the patient requires 36 minutes then the scheduler will block two slots. For example, if this first patient requires 18 minutes, the second patient requires 36 minutes, and the third patient requires 18 minutes, then the first patient will be scheduled at 8:00, the second patient will be at $8: 18$ and the third patient will be at 8:54 since the second patient requires 36 minutes. The 8:36 slot is combined to accommodate the second patient's need and is no longer available. One may feel the scheduled appointment time at 'odd' times (such as 8:18 or 8:26) is difficult for patients to follow and for clinics to implement. Actually, clinics could easily round appointment times to the nearest 5-minute interval [11,36] since the effect is minimal [12]. The schedule will then look like 8:00, 8:20, 8:35, 8:55, 9:10, 9:30, 9:50, and so on. One of the key contributions for the minimum grid approach is the interchangeability as presented in section 3.2. This approach allows a scheduling template to be more flexible to accommodate patients' treatment needs.

\section{CONCLUSIONS}

This paper demonstrates a dynamic approach to designing patient appointment template by determining the minimum grid (length of appointment slot) that optimized the total cost of patient wait time, physician idle time, and overtime. The minimum grid is used for the patient type with the least treatment time. Other patient types will just be scheduled at the length of the integer multiplications of the minimum grid. Unlike other common approaches such as scheduling appointment slots based on the average treatment time of each patient type that does not normally account for the daily appointment demand, the proposed minimum grid system is not only cost-effective but also provides flexibility to appointment scheduling that allows combining slots or splitting a slot to meet patients' needs for an appointment of different patient types 
without increasing patient wait time or physician idle time. Five factors were taken into consideration to demonstrate the effectiveness for different clinic conditions. Among a total of 1125 scenarios, the proposed minimum grid system results in a significant cost savings of at least $15.6 \%$ on average and $49.8 \%$ on maximum in comparison to the base-line approaches. The example of the interchangeability among slots for flexible scheduling also illustrates that combining slots result in a cost saving of at least $15.1 \%$ on average and $42.1 \%$ of maximum and dividing slots result in at least $14.1 \%$ on average and $44.9 \%$ of maximum on cost savings. Even though the example is only for two patient types, it can easily be expanded into more patient types. A case study is provided from a primary care facility to demonstrate the feasibility and how the minimum grid changes with clinic condition. Three primary factors, cost ratio, no-show rate, and treatment time ratio, are identified to impact significantly the minimum grid determination. The challenge now is to actually implement this approach into scheduling systems to further demonstrate its effectiveness and simplicity and address any specific system limitations of any clinics in the model to generalize the approach. This paper provides a unique scheduling approach that is cost-effective without generating additional waiting to patients and physicians. In addition, it improves patient access to care, fully utilizes medical resources, reduces clinic cost, and may ultimately enhances quality of care.

\section{CONFLICT OF INTEREST}

The authors indicated no potential conflicts of interest.

\section{NOMENCLATURE}

$\begin{array}{ll}A & \text { Actual appointment starting time } \\ c & \text { Cost } \\ \text { CR } & \text { Cost ratio } \\ \text { CV } & \text { treatment time variation/coefficient of variation } \\ \text { D } & \text { Demand } \\ \text { DB } & \text { Double block scheduling method } \\ D F & \text { Degree of freedom } \\ \text { DS } & \text { Dome scheduling method } \\ E & \text { Expected clinic finish time } \\ F & \text { Appointment finishing time } \\ \text { FU } & \text { Follow-up patients } \\ G & \text { Probability distribution } \\ m & \text { Total number of patient group } \\ M S & \text { Mean square } \\ n & \text { Total number of appointment slots per clinic session } \\ \text { NP } & \text { New patients } \\ \text { NS } & \text { No-show rate } \\ O & \text { clinic overtime } \\ \text { OV } & \text { Office visit patients } \\ P & \text { Physician idle time }\end{array}$




$\begin{array}{ll}\text { PE } & \text { Physical exam } \\ \text { PS } & \text { The proposed scheduling method } \\ q & \text { Total number of patients scheduled in a time slot } \\ r & \text { No-show rate } \\ \text { RV } & \text { Return visit patients } \\ S & \text { Designated appointment starting time } \\ \text { SB } & \text { Single block scheduling method } \\ \text { SI } & \text { Single block scheduling method with an initial double book } \\ \text { SS } & \text { Sum of square } \\ T & \text { Physician treatment time } \\ \text { TR } & \text { Treatment time ratio } \\ W & \text { Patient wait time } \\ \text { WCC } & \text { Well child care } \\ X & \text { Scheduled time interval of an appointment slot }\end{array}$

Greek

$\alpha$

Statistical significant level

$\mu \quad$ Mean/average

$\sigma \quad$ Standard deviation

$\begin{array}{ll}\text { Subscripts } & \\ i & \text { Index number for } n \\ j & \text { Index number for } m \\ k & \text { Index number for } p\end{array}$

REFERENCES

[1] Gupta D, Denton B. Appointment scheduling in health care: Challenges and opportunities. IIE Transactions. 2008, 40(9):800-819

[2] Cayirli T, Veral E, Rosen H. Designing appointment scheduling systems for ambulatory care services. Health Care Management Science. 2006, 9(1):47-58

[3] Robinson LW, Chen RR. Scheduling doctor's appointments: optimal and empirically-based heuristic policies. IIE Transactions. 2003, 35(3):295-307

[4] Tsai PJ, Teng G. A stochastic scheduling system on multiple resources with dynamic call-in sequence and patient no-shows for an outpatient clinic. European Journal of Operational Research. 2014, 239(2):427-435

[5] Millhiser WP, Veral EA, Valenti BC. Assessing appointment systems' operational performance with policy targets. IIE Transactions on Healthcare Systems Engineering. 2012, 2(4):274-289

[6] Hahn-Goldberg S, Carter MW, Beck JC, Trudeau M, Sousa P, Beattie K. Dynamic optimization of chemotherapy outpatient scheduling with uncertainty. Health Care Management Science. 2014, DOI 10.1007/s10729-014-9268-0

[7] Erdogan SA, Denton B. Dynamic Appointment Scheduling of a Stochastic Server with Uncertain Demand. INFORMS Journal on Computing. 2013, 25(1):116-132

[8] Hong TS, Shang PP, Arumugam M, Yusuff RM. Use of simulation to solve outpatient clinic problems: A review of the literature. South African Journal of Industrial Engineering. 2013, 24(3):27-42

[9] Denton BT, Rahman AS, Nelson H, Bailey AC. Simulation of a multiple operating room surgical suite. Proceedings of the $38^{\text {th }}$ conference on Winter simulation. 2006, Dec. 3-6, Monterey, CA, USA:1-14 
[10] Cayirli T, Veral E. Outpatient Scheduling in Health Care: A Review of Literature. Production Operations Management. 2003, 12(4):519-549

[11] Wu X, Khasawneh M, Yue D, Chu Y, Gao Z. A simulation study of outpatient scheduling with multiple providers and a single device. International Journal of Computational Intelligence Systems. 2014, 7(2): $15-25$

[12] Badri M, Hollingsworth J. A Simulation Model for Scheduling in the Emergency Room. International Journal of Operations \& Production Management. 1993, 13(3):13-24

[13] O'Brien-Pallas L, Baumann A, Donner G, Lochhaas-Gerlach J, Luba M. Forecasting models for human resources in health care. Journal of Advanced Nursing. 2001, 33(1):120-129

[14] Berg BP, Denton BT, Erdogan SA, Rohleder T. Optimal booking and scheduling in outpatient procedure centers. Computers \& Operations Research. 2014, 50:24-37

[15] Lotfi V, Torres E. Improving an outpatient clinic utilization using decision analysis-based patient scheduling. Socio-Economic Planning Sciences. 2014, 48(2):115-126

[16] Klassen KJ, Yoogalingam R. Appointment system design with interruptions and physician lateness. International Journal of Operations \& Production Management. 2013, 33(4):394-414

[17] Donnellan F, Hussain T, Aftab AR, McGurk C. Reducing unnecessary outpatient attendances. International Journal of Health Care Quality Assurance. 2010, 23(5):527-531

[18] Ho C, Lau H. Minimizing Total Cost in Scheduling Outpatient Appointments. Management Science. 1992, 38(12):1750-1764

[19] Patrick J, Puterman ML, Queyranne M. Dynamic Multipriority Patient Scheduling for a Diagnostic Resource. Operations Research. 2008, 56(6):1507-1525

[20] Dexter F, Blake JT, Penning DH, Sloan B, Chung P, Lubarsky DA. Use of Linear Programming to Estimate Impact of Changes in a Hospital's Operating Room Time Allocation on Perioperative Variable Costs. Anesthesiology. 2002, 96(3):718-724

[21] Huang Y, Hancock W, Herrin G. An Alternative Outpatient Scheduling System: Improving the Outpatient Experience. IIE Transactions on Healthcare Systems Engineering. 2012, 2(2):97-111

[22] Oh H, Muriel A, Balasubramanian H, Atkinson K, Ptaszkiewicz T. Guidelines for scheduling in primary care under different patient types and stochastic nurse and provider service times. IIE Transactions on Healthcare Systems Engineering. 2013, 3(4):263-279

[23] Huang Y. Ancillary service impact on outpatient scheduling. International Journal of Health Care Quality Assurance. 2013, 26(8):746-759

[24] Chen RR, Robinson LW. Sequencing and Scheduling Appointments with Potential Call-In Patients. Production and Operations Management. 2014, DOI: 10.1111/poms.12168

[25] Cayirli T, Yang KH, Quek SA. A Universal Appointment Rule in the Presence of No-Shows and WalkIns. Production and Operations Management. 2012, 21(4):682-697

[26] Taner MT, Sezen B. An assessment of diagnostic efficiency by Taguchi/DEA methods. International Journal of Health Care Quality Assurance. 2009, 22(1):93-98

[27] Kopach R, DeLaurentis PC, Lawley M, Muthuraman K, Ozsen L, Rardin R, Wan H, Intrevado P, Qu X, Willis D. Effects of clinical characteristics on successful open access scheduling. Health Care Management Science. 2007, 10(2):111-124

[28] O'Connor ME, Matthews BS, Gao D. Effect of Open Access Scheduling on Missed Appointments, Immunizations, and Continuity of Care for Infant Well-Child Care Visits. Archives of Pediatrics \& Adolescent Medicine. 2006, 160(9):889-893

[29] Bundy DG, Randolph GD, Murray M, Anderson J, Margolis PA. Open Access in Primary Care: Results of a North Carolina Pilot Project. Pediatrics. 2005, 116(1):82-87

[30] Forjuoh SN, Averitt WM, Cauthen DB, Couchman GR, Symm B, Mitchell M. Open-Access Appoinment Scheduling in Family Practice: Comparison of a Demand Prediction Grid With Actual Appointments. Journal of the American Board of Family Practice. 2001, 14(4):259-265 
[31] Peng Y, Qu X, Shi J. A hybrid simulation and genetic algorithm approach to determine the optimal scheduling templates for open access clinics admitting walk-in patients. Computers \& Industrial Engineering. 2014, 72:282-296

[32] Zeng B, Zhao H, Lawley M. The impact of overbooking on primary care patient no-show. IIE Transactions on Healthcare Systems Engineering. 2013, 3(3):147-170

[33] Brunner JO, Bard JF, Kolisch R. Flexible shift scheduling of physicians. Health Care Management Science. 2009, 12(3):285-305

[34] Dexter F, Macario A, Traub RD, Hopwood M, Lubarsky DA. An operating room scheduling strategy to maximize the use of operating room block time: computer simulation of patient scheduling and survey of patients' preferences for surgical waiting time. Anesthesia \& Analgesia. 1999, 89(1):7-20

[35] Isken MW. An Implicit Tour Scheduling Model with Applications in Healthcare. Annals of Operations Research. 2004, 128(1-4):91-109

[36] Vermeulen IB, Bohte SM, Elkhuizen SG, Lameris H, Bakker PJM, La Poutre H. Adaptive resource allocation for efficient patient scheduling. Artificial Intelligence in Medicine. 2009, 46(1):67-80

[37] Van Zon AH, Kommer GJ. Patient flows and optimal health-care resource allocation at the macrolevel: a dynamic linear programming approach. Health Care Management Science. 1999, 2(2):87-96

[38] Augusto V, Xie X, Perdomo V. Operating theatre scheduling with patient recovery in both operating rooms and recovery beds. Computers \& Industrial Engineering. 2010, 58(2):231-238

[39] Kaandorp GC, Koole G. Optimal outpatient appointment scheduling. Health Care Management Science. 2007, 10(3):217-229

[40] Huang Y, Kammerdiner A. Reduction of Service Time Variation in Patient Visit Groups Using Decision Tree Method for an Effective Scheduling. International Journal of Healthcare Technology and Management. 2013, 14(1/2):3-21

[41] Lamiri M, Xie X, Dolgui A, Grimaud F. A stochastic model for operating room planning with elective and emergency demand for surgery. European Journal of Operational Research. 2008, 185(3):1026-1037

[42] Huang Y, Marcak J. Radiology scheduling with consideration of patient characteristics to improve patient access to care and medical resource utilization. Health Systems. 2013, 2(2):93-102

[43] Klassen KJ, Rohleder TR. Scheduling Outpatient Appointments in a Dynamic Environment. Operations Managment. 1996, 14(2):83-101

[44] May JH, Strum DP, Vargas LG. Fitting the Lognormal Distribution to Surgical Procedure Times. Decision Sciences. 2000, 31(1):129-148

[45] Yang KK, Lau ML, Quek SA. A New Appointment Rule for a Single-Server, Multiple-Customer Service System. Naval Research Logistics. 1998, 45(3):313-326

[46] Huang Y, Zuniga P. Dynamic Overbooking Scheduling System to Improve Patient Access. Journal of the Operational Research Society. 2012, 63(6):810-820 


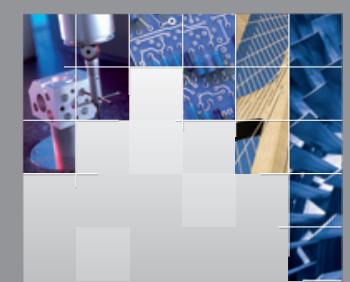

\section{Enfincering}
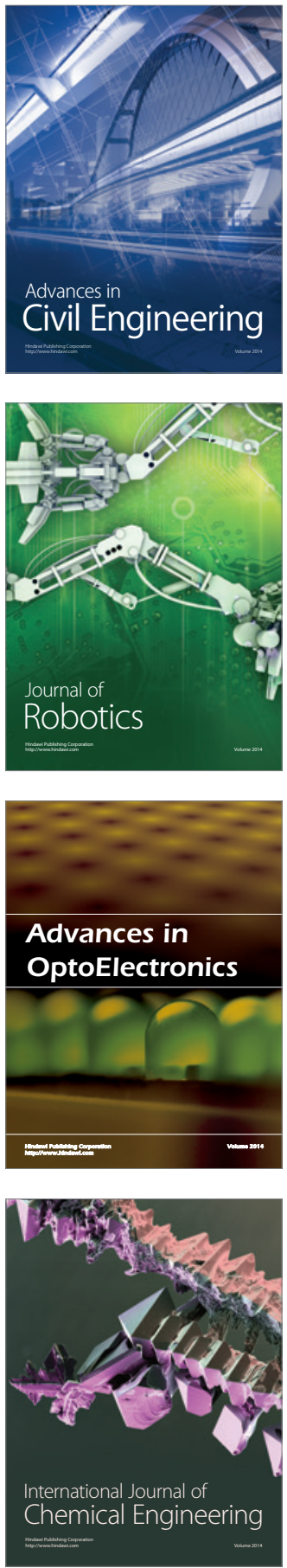

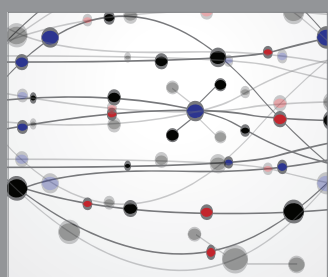

The Scientific World Journal

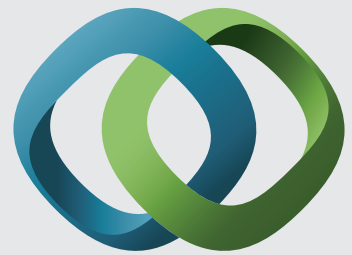

\section{Hindawi}

Submit your manuscripts at

http://www.hindawi.com
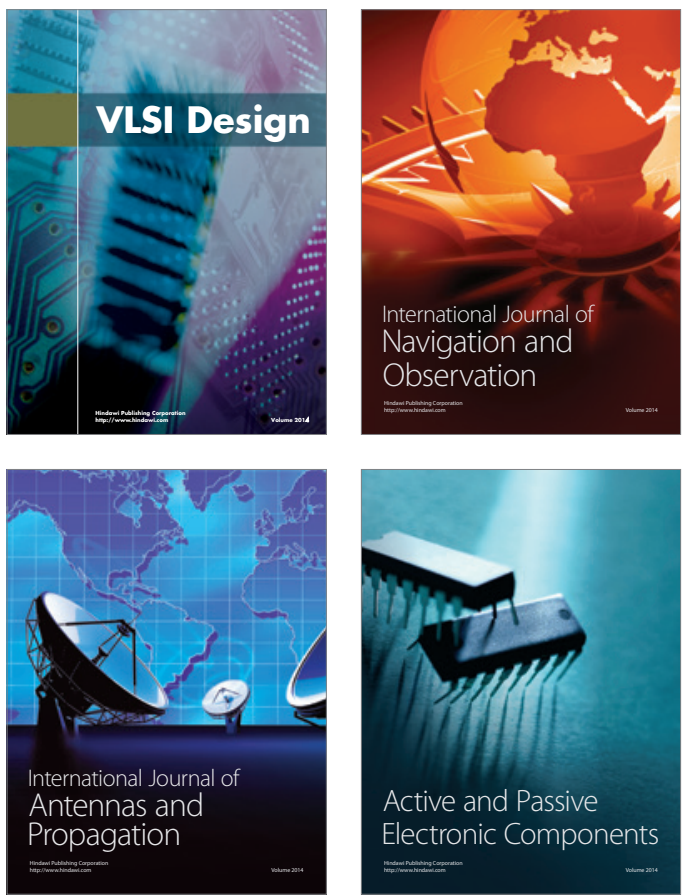
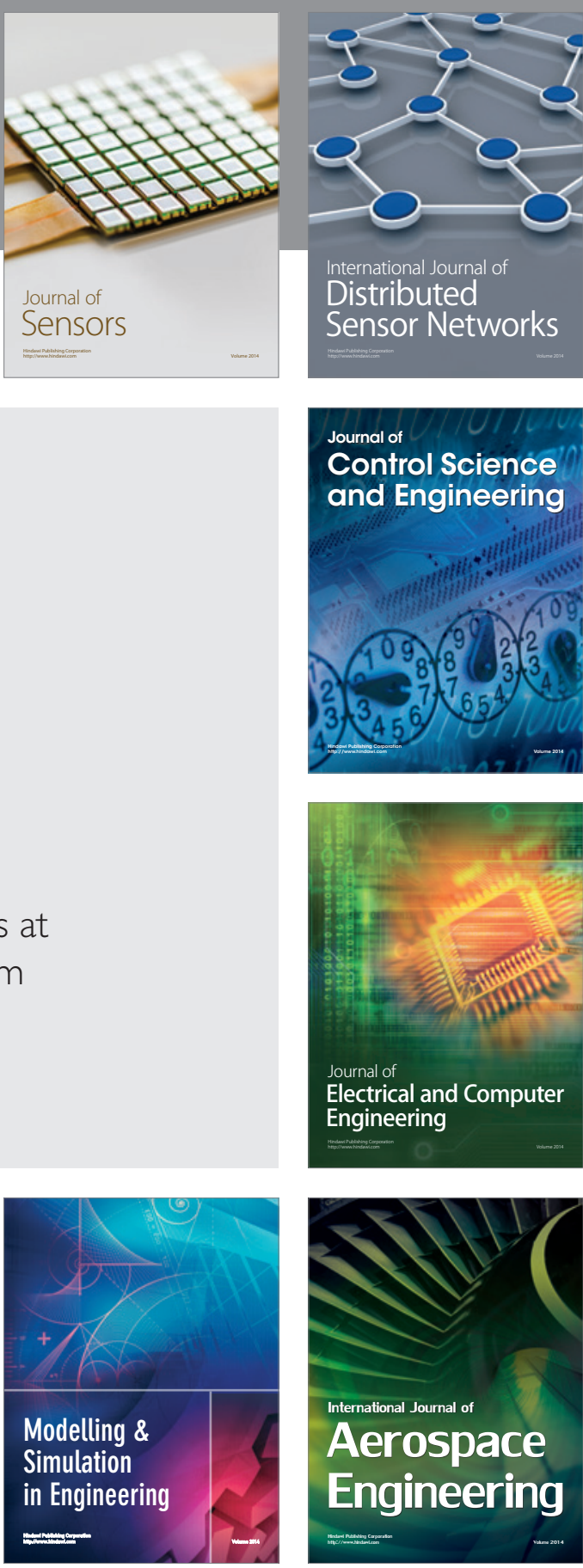

International Journal of

Distributed

Sensor Networks

Journal of

Control Science

and Engineering
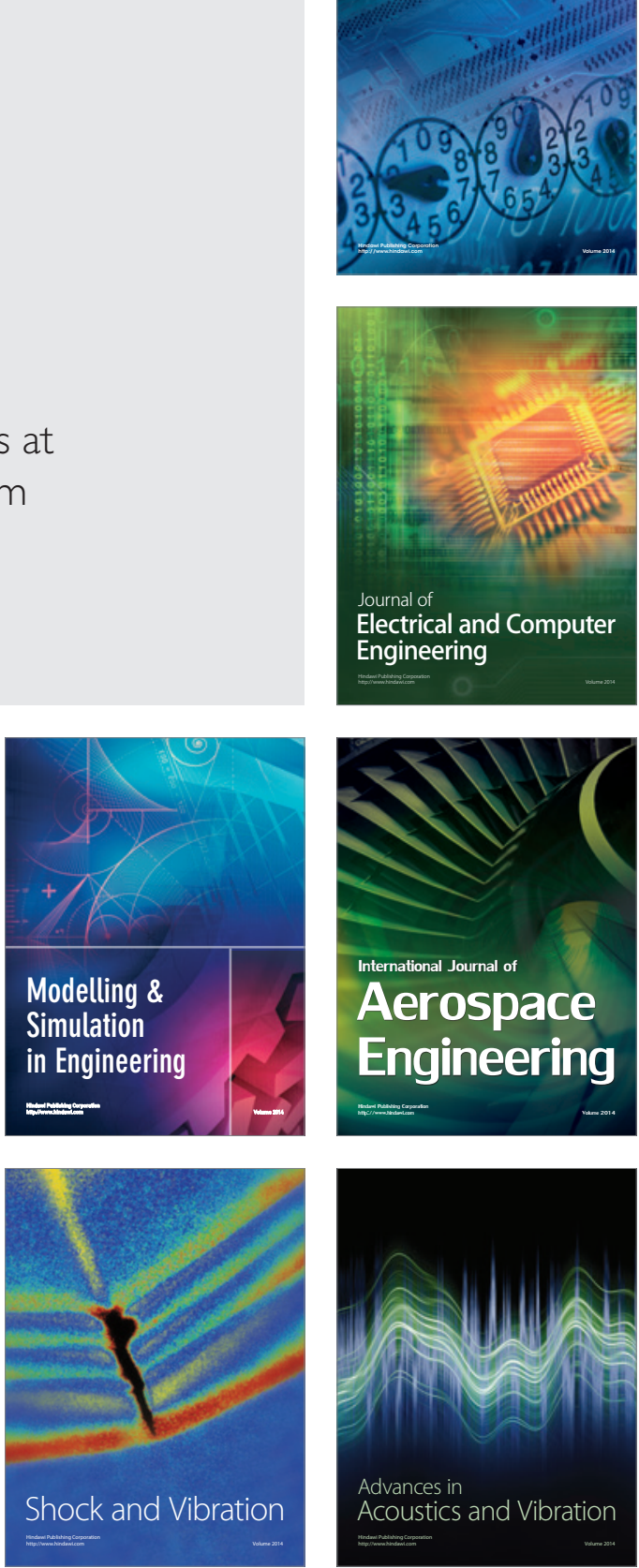